\title{
Transcriptomic analysis revealed the mechanism of oil dynamic accumulation during developing Siberian apricot (Prunus sibirica L.) seed kernels for the development of woody biodiesel
}

Jun Niu ${ }^{1+}$, Jiyong An ${ }^{1+}$, Libing Wang ${ }^{2}$, Chengliang Fang ${ }^{3+}$, Denglong Ha ${ }^{3}$, Chengyu Fu ${ }^{4}$, Lin $\mathrm{Qiu}^{3}$, Haiyan $\mathrm{Yu}^{2}$, Haiyan Zhao ${ }^{3}$, Xinyu Hou', Zheng Xiang ${ }^{1}$, Sufan Zhou' ${ }^{1}$ Zhixiang Zhang ${ }^{1}$, Xinyi Feng ${ }^{1}$ and Shanzhi Lin ${ }^{*}$

\begin{abstract}
Background: Siberian apricot (Prunus sibirica L.) has emerged as a novel potential source of biodiesel in China, but the molecular regulatory mechanism of oil accumulation in Siberian apricot seed kernels (SASK) is still unknown at present. To better develop SASK oil as woody biodiesel, it is essential to profile transcriptome and to identify the full repertoire of potential unigenes involved in the formation and accumulation of oil SASK during the different developing stages.

Results: We firstly detected the temporal patterns for oil content and fatty acid (FA) compositions of SASK in 7 different developing stages. The best time for obtaining the high quality and quantity of SASK oil was characterized at 60 days after flowering (DAF), and the representative periods (10,30,50,60, and 70 DAF) were selected for transcriptomic analysis. By Illumina/Solexa sequencings, approximately 65 million short reads (average length $=96$ bp) were obtained, and then assembled into 124,070 unigenes by Trinity strategy (mean size $=829.62$ bp). A total of $3,000,2,781,2,620$, and 2,675 differentially expressed unigenes were identified at 30, 50, 60, and 70 DAF (10 DAF as the control) by DESeq method, respectively. The relationship between the unigene transcriptional profiles and the oil dynamic patterns in developing SASK was comparatively analyzed, and the specific unigenes encoding some known enzymes and transcription factors involved in acetyl-coenzyme A (acetyl-CoA) formation and oil accumulation were determined. Additionally, 5 key metabolic genes implicated in SASK oil accumulation were experimentally validated by quantitative real-time PCR (qRT-PCR). Our findings could help to construction of oil accumulated pathway and to elucidate the molecular regulatory mechanism of increased oil production in developing SASK.
\end{abstract}

Conclusions: This is the first study of oil temporal patterns, transcriptome sequencings, and differential profiles in developing SASK. All our results will serve as the important foundation to further deeply explore the regulatory mechanism of SASK high-quality oil accumulation, and may also provide some reference for researching the woody biodiesel plants.

Keywords: Siberian apricot, Woody biodiesel, Transcriptome sequencing, Differential expression, Oil accumulation mechanism

\footnotetext{
* Correspondence: szlin@bjfu.edu.cn

${ }^{\dagger}$ Equal contributors

${ }^{1}$ College of Biological Sciences and Biotechnology, College of Nature

Conservation, National Engineering Laboratory for Tree Breeding, Key

Laboratory of Genetics and Breeding in Forest Trees and Ornamental Plants,

Ministry of Education, Beijing Forestry University, Beijing 10083, China

Full list of author information is available at the end of the article
}

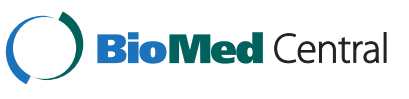

(C) 2015 Niu et al.; licensee BioMed Central. This is an Open Access article distributed under the terms of the Creative Commons Attribution License (http://creativecommons.org/licenses/by/4.0), which permits unrestricted use, distribution, and reproduction in any medium, provided the original work is properly credited. The Creative Commons Public Domain Dedication waiver (http://creativecommons.org/publicdomain/zero/1.0/) applies to the data made available in this article, unless otherwise stated. 


\section{Background}

With the increasing energy demand and environmental pollution problems caused by the fossil fuels, the renewable and clean of energy sources have become an inevitable choice for sustainable development of society and economy. Biodiesel, an alternative diesel fuel, was produced from biological sources such as plant oils or animal fats and has become a popular and environmentfriendly fuel with renewability, low-emissions, security, and biodegradability [1]. However, the high cost of biodiesel has been the major barrier to its commercialization, and the application of edible oils to biodiesel production would lead to the problem of food versus fuel $[2,3]$. Recently, woody seed oils with a notable advantage over conventional feedstocks have been used as potential raw materials for biodiesel production $[2,3]$. Thus, it is important to develop woody resources for biodiesel. In our previous study, Siberian apricot has been characterized as a novel potential raw material for biodiesel from the ten candidate oleiferous tree species [3].

Siberian apricot (Prunus sibirica L.), a member of the family Rosaceae and the genus Prunus, is widely distributed in the temperate and mountainous districts of northern and northeastern China, eastern and southeastern regions of Mongolia, eastern Siberia regions, and maritime territory of Russia [4]. In China, Siberian apricot is one of the most economically and ecologically important and intensively studied tree species owing to its very plentiful resource, superior adaptability, and ecological benefits [5,6]. Moreover, the annual production of Siberian apricot seeds is above 192,500 tons and the average yield of seed oils is approximately 0.8 ton/ha in China [7]. Recently, the oils of SASK have been used in many fields, such as edible oils, lubricants, cosmetics, and surfactants, especially in biodiesel feedstocks $[5,6,8]$. Based on our evaluations of 17 selected Siberian apricot germplasms (oil content ranged from $44.73 \%$ to $57.83 \%$ ) for biodiesel properties, the best Siberian apricot germplasm for biodiesel production has been identified with a high percentage of oleic $(65.23 \% \pm 4.97 \%)$ and linoleic acid $(28.92 \% \pm 4.62 \%)$, showing the potential for using SASK oil as a new source of biodiesel feedstocks in China [9-11]. Although considerable progress of Siberian apricot have been made in oil contents, FA compositions, biodiesel traits, ecological uses, afforestation, and medicinal utilizations [5,6,8-11], little is known about the molecular regulatory mechanism of oil accumulation in SASK. Thus, the transcriptomic analysis of developing SASK has become an imperative for the development of woody biodiesel.

In recent years, advances in low cost next-generation sequencing technology have made RNA sequencing (RNA-Seq) to become an effective choice for gene expression studies [12]. Although RNA-Seq has been performed on the transcriptomic analysis of many oilseed plants such as soybean, peanut, rapeseed, Ricinus communis, Euonymus alatus, Landoltia punctata, and Tropaeolum majus, and some known enzymes and genes were characterized to be involved in FA and triacylglycerol (TAG) biosynthesis [13-18], up to now reports about this situation only in oil palm, olive, and coconut for woody plants $[19,20]$.

Recently, transcriptomic analysis for different tissues of Siberian apricot has been performed by 454 sequencing, and some candidate unigenes were annotated in oil biosynthesis [21], but the obtained results are not still suitable for us to deeply explore the biochemical and molecular mechanism of oil dynamic accumulation in developing SASK. In this work, the first kinetic patterns of oil contents and FA compositions were detected at different developing stages $(10,20,3040,50,60$, and 70 days after flowering (DAF)), and the optimal periods for high quality and quantity of SASK oils and for comparative deep transcriptomic analysis were determined. The transcriptomes of developing SASK were sequenced by using Illumina technology, and then the assembled unigenes were functionally annotated. Moreover, the differentially expressed genes for the enzymes and transcription factors involved in oil accumulation of developing SASK were screened by DESeq method, and experimental validation of some key genes was performed by qRT-PCR. Overall, the temporal accumulated patterns of oils and FA compositions, and the transcriptional profiles of transcriptional regulatory factors and metabolic enzymes associated with the biosynthesis of pyruvate, acetyl-coenzyme A (acetyl-CoA), FA, TAG, and oil body were systematically analyzed in developing SASK, which will contribute to elucidate the molecular and metabolic mechanisms leading to increased oil biosynthesis and accumulation in SASK.

\section{Results and discussion}

\section{Dynamic changes of oil content and FA compositions in} developing SASK

In general, high oil content is considered to be the vital metric for an energy-efficient plant. The study in different Siberian apricot germplasms has shown that the oil content of the fully ripened SASK, ranged from $44.73 \%$ to $57.83 \%[7,9,10]$, was higher than that of traditional oil plants such as Jatropha curcas (38.09\%) and Sapium sebiferum (12\% to $29 \%$ ) [22], indicating a high commercial value for SASK oils. To explore the dynamic accumulated patterns of oils in developing SASK, we evaluated the SASK oil contents at different developing stages (Figure 1). There was a gradual increase in SASK oil content from 10 DAF $(4.00 \% \pm 0.39 \%)$ to $60 \mathrm{DAF}$ $(50.68 \% \pm 4.18 \%)$, followed by approximately $2 \%$ decline at 70 DAF (fully ripe), indicating that the optimal 


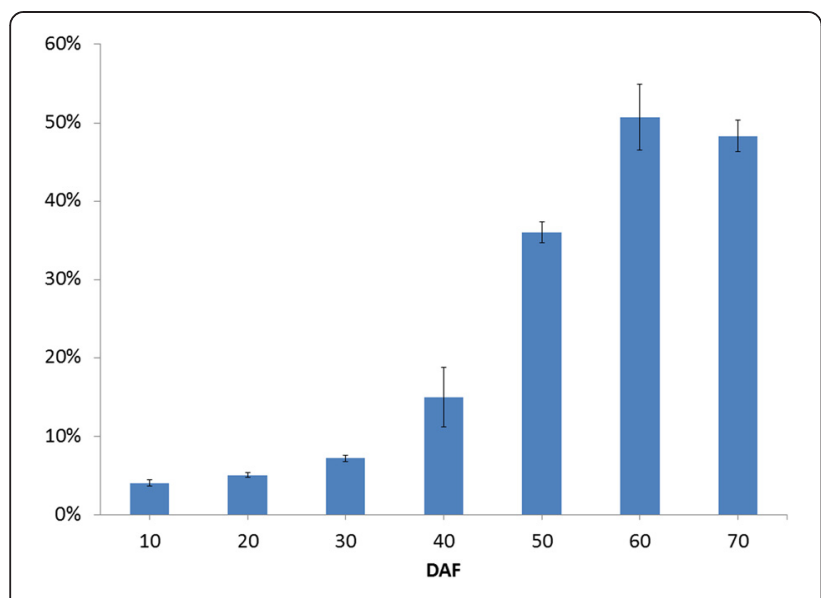

Figure 1 The SASK oil content at different development period.

harvest time for obtaining the maximum SASK oil content was at 60 DAF. It is important to note that the relative proportion of saturated, monounsaturated, and polyunsaturated FAs is the critical factor affecting biodiesel fuel properties [3]. In this study, the eight kinds of FAs were firstly detected in SASK oils at different developing stages, and the temporal patterns of their relative proportions were analyzed (Figure 2). We found that the C18:1 (oleic acid) relative proportion gradually increased from 10 DAF $(34.37 \% \pm 1.57 \%)$ to $70 \operatorname{DAF}(67.41 \% \pm$ $2.35 \%)$ with a remarkable elevated degree at 40 to 50 DAF, and the percentage of C18:2 (linoleic acid) exhibited a peak value $(50.07 \% \pm 2.87 \%)$ only at $30 \mathrm{DAF}$, but almost no significant alteration $(23.29 \%$ to $38.45 \%)$ for the other different developing periods. Additionally, the other saturated or long chain FAs with a lower relative proportion showed a slight modification in developing SASK. Impressively, the total relative proportion of C18:1 and C18:2 in SASK oils ranged from 60.66\% to $91.74 \%$ at 10 to 70 DAF (Figure 2), and therefore a higher ratio of oleic and linoleic acid revealed SASK oils with a high quality as a novel potential woody biodiesel feedstock in China, which corresponded to the previous investigations on SASK oils [7,10].

Taken together, our findings on the highest oil content $(50.68 \% \pm 4.18 \%)$ and the near-maximal total percentage of C18:1 and C18:2 (91.64\%) in SASK at 60 DAF indicated that the very best harvest time for SASK oils with high quality and quantity is at 60 DAF. Moreover, according to the temporal patterns of oil content and the total relative proportion of $\mathrm{C} 18: 1$ and $\mathrm{C} 18: 2$ in developing SASK, the samples from five crucial periods $(10,30$, 50,60 , and 70 DAF) were selected as the experimental materials for comparative deep transcriptomic analysis to better explore the molecular and metabolic regulatory mechanism of SASK oil increased-accumulation.

\section{Illumina sequencing and de novo assembly of developmental SASK}

To clarify a global overview of the gene expressing profiles in developing SASK, a total of five cDNA libraries were constructed from different developing SASK RNA samples and were respectively sequenced by the Illumina transcriptome sequence. More than 5 GB raw data were respectively generated from 5 RNA-Seq samples, and then an average $(55,994,330)$ of short reads were obtained after removing low-quality reads and adaptor sequences (Table 1). The numbers of raw data and short reads were larger in developing SASK than in longan

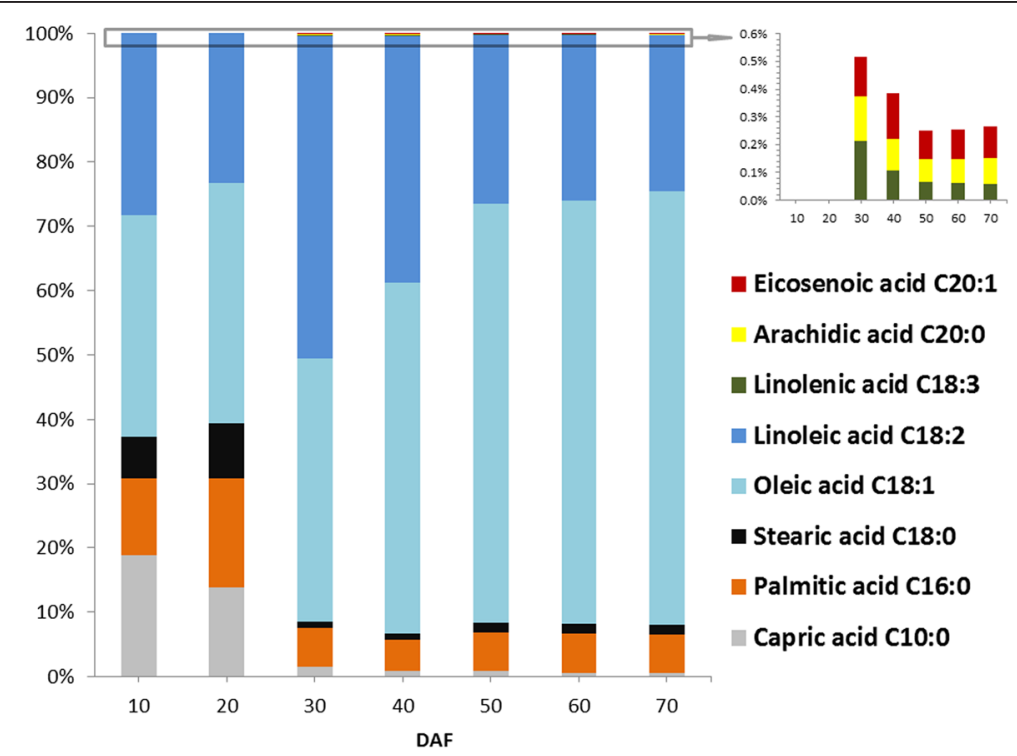

Figure 2 Changes in the fatty acid composition during SASK development. The data represent the mean of three independent measurements. 
[23] and peanut [15], suggesting that SASK Illumina sequencing could construct a complete read database. All high-quality reads were deposited in the NCBI Short Read Archive (SRA) database under accession numbers SRR1564517, SRR1568273, SRR1568275, SRR1568789, and SRR1568805.

At present, there are no standard criteria to evaluate the quality of transcriptome assemblies [24]. To ensure accuracy and reliability of our SASK transcriptome assembly, 500,000 reads randomly selected from all reads were compared with NCBI database of nucleotide for pollution monitoring. From the Figure 3, the first Prunus dulcis, belonging to the same family Rosaceae with our experimental material (P. sibirica L.), was shown to have over three times as many reads as the second Vitis vinifera, indicating the reliability of our Illumina transcriptome sequencing data. Additionally, all these reads obtained from SASK of five developing stages were assembled by the Trinity software [25], and then lowcomplexity and low-quality reads were filtered out, resulting in 226,682 transcripts with GC content of $49.09 \%$. By clustering through the Trinity program, we obtained 124,070 unigenes (N50: 1,603 bp) with the mean length of 829.62 bp (Additional file 1: Table S1) that is longer than those assembled from Centella asiatica (474 bp) [26], Yellow Horn (462 bp) [27], and peanut (751 bp) [15]. We also statistically found that there were 16,698 unigenes (13.46\%) in the length range of 1,000 to $2,000 \mathrm{bp}$ and 13,507 unigenes $(10.88 \%)$ with length more than 2,000 bp (Additional file 2: Figure S1). Thus, a total of 30,205 unigenes $(\geq 1,000 \mathrm{bp})$ in developing SASK, nearly 3 times more than our previous transcriptome analysis for different tissues of Siberian apricot [21], could massively fill and enrich the dataset of Siberian apricot.

Functional annotation and classification of SASK unigenes In order to understand specific functions of all the assembled unigenes in developing SASK, they were predicted via Basic Local Alignment Search Tool (BLAST) with a cut-off E-value of 10 to 5 in NCBI non-redundant protein $(\mathrm{Nr})$ database, Swiss-Prot protein database (SwissProt), Arabidopsis proteome (AP) database, Clusters of Orthologous Groups (COG) database, Gene

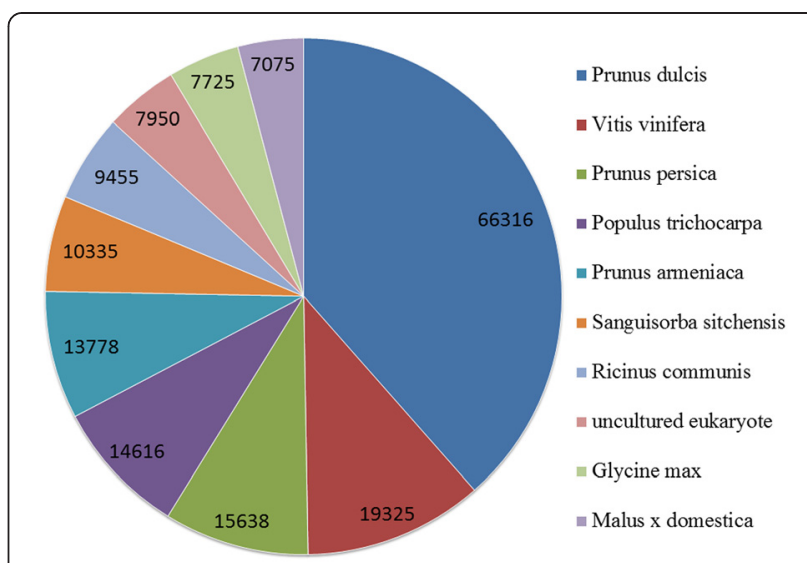

Figure 3 Species distribution of the top BLAST hits for reads in $\mathrm{Nr}$ database.

Ontology (GO), and Kyoto Encyclopedia of Genes and Genomes (KEGG). The BLAST results showed that 47,406 (38.21\%), 30,511 (24.59\%), 46,949 (37.84\%), 17,870 (14.40\%), 36,553 (29.46\%), and 18,807 (15.10\%) unigenes of developing SASK were highly similar with known proteins in Nr, SwissProt, AP, COG, GO, and KEGG databases, respectively (Additional file 3: Table S2 and Additional file 4: Table S3). Of all SASK unigenes, 49,904 (40.22\%) homologous unigenes showed indeed that a considerable functional contig size was obtained. However, those unmatched SASK unigenes $(74,166$, $59.78 \%$ ) might be as the putative tissue-specific novel genes or probably resulted from the shorter sequences with a lack of characterized protein domain to get BLAST hits [27].

The Gene Ontology (GO) annotation for the assembled unigenes was used to categorize the functions of the predicted SASK unigenes [28], and a total of 36,553 unigenes were assigned to the 3 main GO categories and 65 subcategories. Additional file 5: Figure S2 shows that 'metabolic process,' 'cellular process,' 'binding,' and 'catalytic activity' are the most dominant categories involving more than 20,000 unigenes, but only a few of genes were associated with terms such as 'viral reproduction,' 'cell junction,' and 'channel regulator activity'. To further evaluate the completeness of our transcriptomic libraries and the effectiveness of the

Table 1 Raw data and valid data statistics

\begin{tabular}{|c|c|c|c|c|c|c|}
\hline \multirow[t]{2}{*}{ Sample (DAF) } & \multicolumn{2}{|l|}{ Raw data } & \multicolumn{3}{|l|}{ Valid data } & \multirow{2}{*}{$\begin{array}{l}\text { Valid ratio } \\
\text { (reads) (\%) }\end{array}$} \\
\hline & Read & Base & Read & Base & Average length & \\
\hline 10 & $60,940,126$ & $6,094,012,600$ & $53,830,008$ & $5,182,918,614$ & 96.28 & 88.33 \\
\hline 30 & $65,980,058$ & $6,598,005,800$ & $58,413,976$ & $5,636,764,333$ & 96.50 & 88.53 \\
\hline 50 & $64,572,540$ & $6,457,254,000$ & $56,984,496$ & $5,479,436,388$ & 96.16 & 88.25 \\
\hline 60 & $72,078,976$ & $7,207,897,600$ & $57,262,494$ & $5,368,833,289$ & 93.76 & 79.44 \\
\hline 70 & $62,898,984$ & $6,289,898,400$ & $53,480,678$ & $5,112,244,068$ & 95.59 & 85.03 \\
\hline
\end{tabular}


annotation processes, all SASK unigenes were aligned to the COG database to predict and classify by possible function [29]. The resulting 17,870 (14.4\%) unigenes were assigned to the 25 COG classifications (Additional file 6: Figure S3), among which the cluster for 'Posttranslational modification, protein turnover, chaperones' represented the largest group $(3,875,21.68 \%)$ followed by 'Signal transduction mechanisms' $(3,379,18.91 \%)$ and 'General function prediction only' (2,662, 14.90\%). Subsequently, to identify the biological functions and interactions of genes in developing SASK, the KEGG was applied to annotate the reference canonical pathways [30]. In total, 18,807 sequences were assigned to 313 KEGG pathways. Most of them were 'Ribosome' (1,254, $6.67 \%)$, 'Protein processing in endoplasmic reticulum' (604, 3.21\%), 'Oxidative phosphorylation' (534, 2.84\%), 'Glycolysis/gluconeogenesis' (494, 2.63\%), and 'Plantpathogen interaction' (490, 2.61\%). Moreover, we also annotated several signaling networks of reproductive biology, such as 'Non-homologous end-joining' and 'HIF-1 signaling pathway'.

\section{Analysis of differential gene expression for developing SASK}

To fully explore the differential expressions of the specific genes that are actually implicated in developing SASK, the clean reads from each library were mapped to the transcriptomic database for profiling the expressions of unigenes. The utilization rate of reads and the expression rate of unigenes showed that 10 DAF samples had the most unigenes $(91,563,74.28 \%)$ mapped to our constructed unigene database, whereas 50 DAF sample had the least unigenes $(75,667,60.9 \%)$ (Table 2 and Additional file 7: Figure S4). Also, the RPKM values were statistically calculated to select differential unigenes from developing SASK by using the DESeq method [31,32] (Additional file 8: Figure S5 and Additional file 9: Table S4). Our results revealed that 10:30 DAF exhibited the most up-regulated $(1,513)$ and the least downregulated unigenes $(1,487)$, whereas 10:70 DAF had the least up-regulated (726) and the most down-regulated unigenes $(1,949)$ (Figure 4A). By Venn diagram analysis, $838,198,74$, and 90 up-regulated unigenes and 602, 354, 370 , and 295 down-regulated unigenes were identified to be specific expression at 30,50,60, and 70 DAF
(Figure 4B,C), respectively. Thus, combining with the temporal patterns of SASK oil at developing stages (Figure 1), we suggested that the candidate unigenes associated with oil accumulation pathway may be expressed at early stage of developing SASK.

\section{Transcript patterns for enzymes and transporters involved in generating pyruvate for SASK FA synthesis}

Sucrose is known as the most source of carbon, and its major metabolic flux through glycolytic pathway is to provide the large amounts of pyruvate required for de novo FA biosynthesis in oilseed plants $[19,33,34]$. In general, plant glycolysis occurs independently in two subcellular compartments of the cytosol and plastid. ATP-dependent phosphofructokinase (PFK) and pyruvate kinase $(\mathrm{PK})$ are considered as two regulatory enzymes of glycolytic pathway in both cytosol and plastid, while pyrophosphate-dependent phosphofructokinase (PFP) only in cytosol has been characterized as a key enzyme to reversibly catalyze the conversion of fructose6-phosphate to fructose-1,6-bisphosphate $[16,19,35]$. Moreover, the interchange of glycolytic intermediates between cytosol and plastid in plants has been implicated in the highly selective plastid transporters, including glycolipid transporter (GLT1), triose phosphate transporter (TPT), glucose-6-phosphate transporter (GPT2), and phosphoenolpyruvate transporter (PPT1) [19,33,36,37]. It is well known that the glycolytic substrates of hexose phosphates, generated from sucrose in the cytosol, are generally transported by GLT1 or GPT2 for plastidial glycolysis [34]. Recently, the transcripts of the plastid-localized enzymes (PFK and PK) and the transporters (GLT1 and PPT1) exhibited an obvious up-regulation, but PFP remained unchanged during ripening, indicating that plastid glycolysis plays a major role in providing pyruvate for high rates of FA synthesis in oil palm $[34,35]$. However, our transcriptional profiles revealed the stable-high expressions of cytosolic PFK (AT4G26270) and PK (AT3G52990) and the lower expressions of plastidial PFK (AT2G22480) and cytosolic PFP (AT1G12000) in developing SASK (Figure 5), suggesting a greater proportion of hexose to pyruvate flux in cytosol. Also consistent with this conclusion, the decreasing expressions in the translocators of GLT1 (AT2G33470), TPT (AT5G46110), and GPT2 (AT1G61800) were observed on developing SASK. It is, therefore, considered that there is a

Table 2 Statistics of reads and the utilization of unigenes

\begin{tabular}{lllllll}
\hline DAF & Valid data & Map data & Data (\%) & All unigene & Exp unigene & Unigene (\%) \\
\hline 10 & $53,830,008$ & $49,899,224$ & 92.70 & 124,070 & 92,163 & 74.28 \\
30 & $58,413,976$ & $54,258,107$ & 92.89 & 124,070 & 84,912 & 68.44 \\
50 & $56,984,496$ & $53,171,111$ & 93.31 & 124,070 & 76,668 & 61.79 \\
60 & $57,262,494$ & $51,322,910$ & 89.63 & 124,070 & 75,667 & 60.99 \\
70 & $53,480,678$ & $47,986,764$ & 89.73 & 124,070 & 84,030 & 67.73 \\
\hline
\end{tabular}



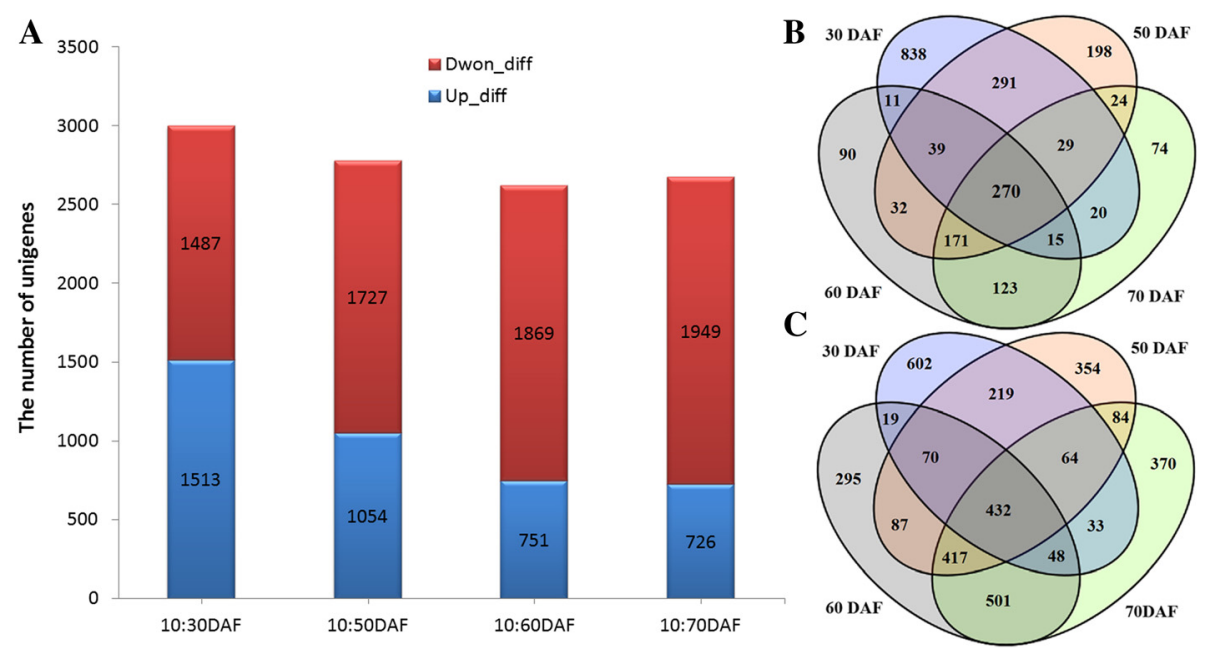

Figure 4 Number and distribution of up-regulated and down-regulated genes. (A) The number of up-regulated or down-regulated unigenes during developing SASK. (B) The distribution of up-regulated genes in differently developmental periods. (C) The distribution of down-regulated genes in differently developmental periods. The detailed sequences were showed in Additional file 9: Table S4.

lower capacity to provide glycolytic substrates (hexose and hexose phosphate) and intermediate (triose phosphate) from cytosol to plastid in developing SASK. Interestingly, an up-regulated expression of the plastidial PK (AT5G52920) and its corresponding PPT1 (AT5G33320) were noted in SASK only at 30 DAF (Figure 5), the period that was important for initial FA biosynthesis (Figure 1). This implies a transportation of PEP from cytosol to plastid and the production of pyruvate required for SASK FA biosynthesis. Together, our results revealed that the cytosolic carbon supply via glycolytic pathway is crucial for de novo FA biosynthesis of developing SASK.

\section{Transcriptional patterns for the enzymes of acetyl-CoA formation for SASK de novo FA biosynthesis}

Acetyl-CoA is an important precursor for de novo FA biosynthesis in plastids, various phytochemical biosynthesis and FA elongation in cytosol, and TCA cycle in mitochondria [38]. However, acetyl-CoA must be synthesized by specific synthetase in each subcellular compartment for a variety of important biosynthesis owing to the impermeability of biological membranes [39], indicating the multiple mechanisms of generating acetylCoA for different acetyl-CoA-requiring metabolisms. To our knowledge, acetyl-CoA synthetase (ACS) and pyruvate dehydrogenase complex (PDC) are thought to be implicated in the conversion of pyruvate to acetyl-CoA for de novo FA biosynthesis in plastids of plants, and four subunits (E1- $\alpha, E 1-\beta, E 2$, and E3) of PDC have been characterized in Arabidopsis [16,39]. In an attempt to determine the importance of the enzymes (ACS and PDC) involved in acetyl-CoA formation for SASK de novo FA biosynthesis, their transcriptional patterns in developing
SASK were analyzed in this study. The SASK homologies of Arabidopsis plastidial PDC E1- $\alpha$ (At1g01090), E1- $\beta$ (At2g34590), E2 (At1g34430), and E3 (At4g16155) were identified as a significantly higher expression at $30 \mathrm{DAF}$, but the homologous gene of ACS (AT5G36880) displayed a lower expression (Figure 5), indicating that plastidial PDC may play an important role in the supply of acetyl-CoA for de novo FA synthesis in developing SASK. Attentively, PDC has also been characterized to locate in mitochondria of plants [40]. Indeed, our annotated four subunits of PDC in developing SASK exhibited high similarity with Arabidopsis mitochondrial E1- $\alpha$ (At1g59900), E1- $\beta$ (At5g50850), E2 (At3g52200), and E3 (At1g48030), but their transcriptional patterns were shown no significant change (Figure 5), which could help to distinguish the PDC distribution between plastids and mitochondria in SASK at transcriptional level. Overall, the generation of acetyl-CoA might be tightly regulated in response of SASK to different developing stages.

\section{Identification of the de novo FA biosynthetic pathway- specific unigenes in developing SASK}

De novo FA synthesis from acetyl-CoA precursors, as the first part of the lipogenesis process, is important in controlling the content and chain length (up to 18 carbons) of the saturated FAs in seed oils. Indeed, it has been reported that a multi-subunit acetyl-CoA carboxylase (ACC, EC: 6.4.1.2), regulated by phosphorylation and redox status as well as PII interactions, is the first committed key enzyme in the de novo FA biosynthesis pathway, and its physiological function is to provide the substrate malonyl-CoA for the biosynthetic saturated FAs [41,42]. Recently, it has been proposed that the intracellular accumulation of free C18:1, 18:1-CoA, and 


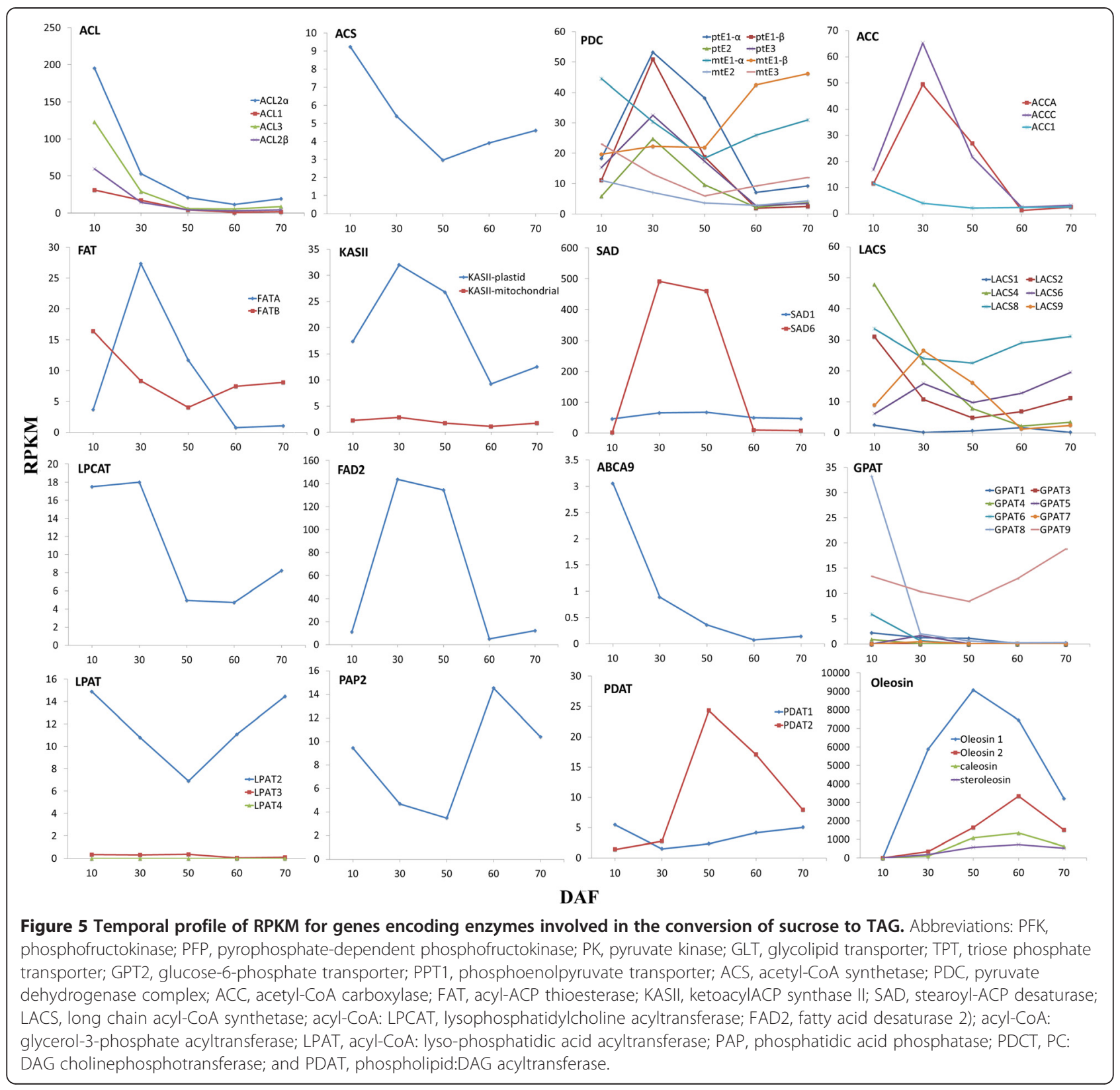

18:1-acyl-carrier protein (ACP) in Brassica napus cellsuspension culture by feeding oleic acid (18:1) Tween esters could result in feedback inhibition of plastidial ACC activity, leading to a reduction of FA synthesis [43]. To characterize whether the transcriptional expressions of plastidial ACCs were linked to the FA biosynthesis in developing SASK, we performed a concurrent analysis of functional annotations and differential profiles for all the obtained unigenes. Here, a total of 10 SASK unigenes were characterized to be homologous with ACC subunits of other species, including 6 Arabidopsis ACC carboxyl transferase subunit alpha (ACCA), 2 Arabidopsis ACC1, and 2 Populus trichocarpa ACC biotin carboxylase subunit (ACCC), but only 2 homologies of $a c c A$ and $a c c C$ exhibited the highest expressing levels at 30 DAF followed by a dramatic decrease at 50 to 60 DAF (Figure 5). Combined with our observations on an obvious increase of FAs (C18:1 is one major component) at $50 \mathrm{DAF}$ and the decreasing rate of FA biosynthesis after 50 DAF (Figures 1 and 2), we considered that a lower expressions of acc genes might be related with a high intracellular accumulation of FAs in developing SASK. Thus, our results prompt us to believe that an inhibitory expression of $a c c$ genes may be at 50 DAF, giving rise to a declined capacity of ACC to generate malonyl-CoA for de novo FA biosynthesis. However, 
further research is needed in transcriptional regulatory mechanism for plastidial $a c c$ as the target gene in de novo FA synthesis of developing SASK.

Before entering the synthesis pathway of FAs, malonylCoA from Acetyl-CoA is firstly transferred to malonylACP by malonyl-CoA-ACP transferase (MAT, EC: 2.3.1.39) to provide two-carbon unit at each step of elongation. There are four reactions of condensation, reduction, and dehydration, and reduction occurred in each synthesized cycle, which is catalyzed by a series of regulatory enzymes. Our results of functional annotations revealed that one MAT, three 3-ketoacyl ACP synthase II (KASII, EC: 2.3.1.179), one 3-ketoacyl ACP synthase III (KASIII, EC: 2.3.1.180), two 3-ketoacyl ACP reductase (KAR, EC: 1.1.1.100), and one enoyl-ACP reductase (EAR, EC: 1.3.1.9) involved in FA preliminary synthesis in developing SASK. After seven elongated cycles, the production of saturated $\mathrm{C} 16: 0-\mathrm{ACP}$ can either be converted to free $\mathrm{C} 16: 0$ by the fatty acyl-ACP thioesterase B (FATB, EC:3.1.2.14 3.1.2.-) or further elongated by KASII to 18:0-ACP. Subsequently, 18:0-ACP can be hydrolyzed to free C18:0 by FATB or be desaturated to $18: 1-\mathrm{ACP}$ by $18: 0-\mathrm{ACP}$ desaturase (SAD, EC:1.14.19.2), and then 18:1-ACP is hydrolyzed to free $\mathrm{C} 18: 1$ by the fatty acyl-ACP thioesterase A (FATA, EC:3.1.2.14 3.1.2.-) [44]. In plants, the relative proportion of FAs was remarkably altered by manipulation of any of these four enzymes (FATA, FATB, SAD, and KASII), but FATA and FATB have shown to be not directly involved in FAs biosynthesis [45,46]. In this study, the $f a t B$ genes with a stable expression were observed in developing SASK, while the fatA genes were notably upregulated only at 30 DAF (Figure 5), suggesting that the resulting free $\mathrm{C} 18: 1$ rather than $\mathrm{C} 16: 0$ and $\mathrm{C} 18: 0$ may be as one main product of plastid FA synthesis in developing SASK, which was coincide with our investigations on FA compositions (Figure 2). Additionally, a total of six kasII unigenes were found in our experiments, where 1 mitochondria kasII unigene with expressive stability and 5 plastid kasII unigenes with a high expression were identified at 30 DAF (Figure 5). It is interesting to note that 2 sad6 genes were sharply up-regulated over 200 folds at 30 and $50 \mathrm{DAF}$, whereas 2 highly-expressed $s a d 1$ genes remained stable in developing SASK (Figure 5). Thus, we considered that the increasing trend of C18:1 (oleic acid) content paralleled to the temporally transcriptional patterns for sad6 and kasII in developing SASK (Figures 2 and 5) could explain SAD6 and KASII as the key enzymes for oleic acid formation in developing SASK. This also was strongly supported by the fact that a reduction in the activities of KASII and SAD caused by antisense gene silencing could lead to a decrease of 18:1 accumulation in Brassica and Arabidopsis seeds [47,48]. In conclusion, 18:1-ACP, as a major metabolite of FA biosynthesis, may be responsible for transportation of FA from plastid to cytosol in developing SASK.
Determination of the unigenes-regulated polyunsaturated FA and TAG biosynthesis in developing SASK

The synthesized free FAs by the FATA/B thioesterases are exported from plastid to cytosol and then converted to the fatty acyl-CoAs by long-chain acyl CoA synthetase (LACS), which can provide the substrate for esterification in endoplasmic reticulum (ER) [49]. In this study, we observed a variety of kinetic transcriptional profiles for lacs in developing SASK. For example, lacs4 and lacs 2 were up-regulated at 10 and $30 \mathrm{DAF}$, and two lacs6 genes displayed high expression at 60 and 70 DAF, but lacs 8 showed stable expression during the whole developing periods, (Figure 5). It is concluded that multigene lacs genes may compensate for the lack of function each other in developing SASK, which could be evidenced by the fact that double (lacs1 and lacs9) or triple (lacs1, lacs8, and lacs9) lacs mutant notably affect the FA content of Arabidopsis seeds [50].

For polyunsaturated FAs synthesis, C18:1 must be esterified by acyl-CoA: lysophosphatidylcholine acyltransferase (LPCAT) from cytosol to phosphatidylcholine (PC) pool in ER and then desaturated by omega-6 FA desaturase (FAD2) in PC pool [51]. In this work, four highly expressed lpcat genes at 10 to $30 \mathrm{DAF}$ and two drastically up-regulated fad2 genes at 30 to 50 DAF were detected in developing SASK (Figure 5), indicating that the polyunsaturated FAs may be as one of main components in SASK oil, which was also experimentally demonstrated on an accumulation of linolenic acid as the secondary main component in developing SASK (Figure 2). It is generally accepted that acyl-CoAs must be transported from the cytosol to ER for glycerolipid synthesis through diffusion by soluble carriers or by some more efficient inter-membrane transporters [44]. Recently, an ABC transporter (AtABCA9) has been identified to be involved in the delivery of FA to the ER in Arabidopsis [52]. However, SASK transcriptional profiles revealed that five unigenes encoding $\mathrm{ABC}$ transporter displayed a lower expression in developing SASK, of which two unigenes (comp63523_c1 and comp67480_c0) showed highly homologous with AtABCA9 transporter protein (Figure 5). Therefore, how this $A B C$ transport system influence FAs transport in developing SASK remains to be further investigated.

The de novo formation of TAG occurs in ER via the Kennedy pathway, in which glycerol-3-phosphate (G3P) is initially acylated to produce TAG by the sn-G3P acyltransferase (GPAT, EC: 2.3.1.15) [53]. The previous studies of Arabidopsis GPATs showed that GPAT1 and GPAT4-8 with $s n-2$ regiospecificity were not required for TAG synthesis, and GPAT9 with $s n-1$ regiospecificity might be involved in TAG synthesis, but the function of GPAT2-3 with undetermined regioselectivity remained unknown [54,55], suggesting a functional divergence of 
the members in GPAT family. In this study, 2 genes of gpat 9 with a high and stable expression and 11 genes of gpat1 and gpat3-7 with an ultralow expression were observed at different developing stages, but 2 genes of gpat8 with specific up-regulation were only found at 10 DAF (Figure 5), indicating the difference of transcriptional expressions for the members of gpat multigene family in developing SASK. This investigation allowed us to speculate that the expressions of gpat multigene family may be tightly regulated in response to developing SASK. Moreover, together with the dynamic accumulated patterns of oils in developing SASK (Figure 1), we concluded that the GPAT9 may function as a key initial acyltransferase for SASK TAG assembly.

In the second step of de novo TAG assembly, lysophosphatidyl acyltransferase (LPAT, EC: 2.3.1.51) and phosphatidic acid phosphohydrolases (PAP, EC: 3.1 .3 .4 ) play the essential roles in the formation of phosphatidic acid (PA) and in the removal of the phosphate group from PA to form diacylglycerol (DAG), respectively. Also, several isozymes of LPAT and PAP have been demonstrated in higher plants $[16,19,56-58]$. Overexpression of rapeseed microsomal LPAT isozymic gene (BAT1.13 or $B A T 1.5)$ could result in an increase in oil content of Arabidopsis seeds [56], but oil biosynthesis was not significantly affected by double knockout of two PAP genes (PAH1 and PAH2) [57]. In order to assess the contribution of LPAT and PAP to DAG biosynthesis in developing SASK, the transcriptional patterns for the homologies of LPATs and PAPs were comparatively analyzed. Here, one LPAT homologous gene lpat2 (AT3G57650) and four PAP homologous genes of pah1 (At3g09560), pah2 (AT5G42870), lpp $\delta$ (AT3G58490), and lpp3 (At3g02600) were characterized with an obvious increasing expression at 50 to 60 DAF (Figure 5), and therefore they are likely to be responsible for DAG biosynthesis in developing SASK. Recently, genetic analysis revealed that mutation of Arabidopsis rod1 (encoding PC:DAG cholinephosphotransferase, PDCT) resulted in a marked decrease in polyunsaturated FAs (18:2 and 18:3) and a concomitant increase in 18:1 in seed, suggesting that PDCT may play an important role in the transfer of 18:1 into PC pool for desaturation and also in the reverse transfer of 18:2 and 18:3 into the TAG synthesis pathway in developing seeds [59]. In this work, the SASK homologies for PDCT in Arabidopsis (At3g15820) showed a much lower expression at 50 to 60 DAF (Figure 6). Thus, the contribution of PDCT to the accumulation of polyunsaturated FAs was very limited in developing SASK, which was in accordance with our detective results of FA compositions (Figure 2).

For the last step of the Kennedy pathway, multiple acyl-CoA:DAG acyltransferase (DGAT) enzymes have been demonstrated for the formation of TAG from DAG and Acyl-CoA in plants. For example, DGAT1, DGAT2, and DGAT3 are responsible for TAG production in Arabidopsis, tung tree, and peanut [60-62]. We annotated all the unigenes of developing SASK in this work. Surprisingly, only two dgat1 genes were incrementally expressed in developing SASK, suggesting that DGAT1 might be one major enzyme specific to TAG biosynthesis of SASK. It was also noted that plant TAG synthesis could be catalyzed by phospholipid: diacylglycerol acyltransferase (PDAT) using the FAs from the PC pool [63]. We found two pdat1 genes with a stable expression during the developing stages and two pdat 2 genes with a high expression at 50 to 60 DAF (Figure 5). Interestingly, the transcriptional level of $p$ dat 2 was temporally paralleled to oil accumulation in developing SASK (Figure 1), implying that PDAT2 may play a major role in TAG synthesis of developing SASK.

Taken together, our investigations could help to characterize which isoforms of these large gene families are mainly responsible for SASK TAG biosynthesis, and also attested to the fact that Siberian apricot possesses many specific enzymes in TAG assembly (Figure 6), providing the important references for the study of lipogenesis process in woody oil trees.

\section{The transcriptionally temporal comparisons of oil body genes in developing SASK}

TAGs can be stored as a form of oil bodies surrounded by a phospholipid monolayer and abundant amphipathic proteins such as oleosin, caleosin, and steroleosin in mature seeds $[16,19,64]$. By the transcriptionally temporal comparisons, we characterized the homologous oleosin-1 and oleosin-2 of Arabidopsis (AT4G25140 and AT5G40420), caleosin of Ficus pumila var (ABV72237.1), and steroleosin of Sesamum indicumm (AAM46847.1) with an increasing or stable-high RPKM values in developing SASK (Figure 5). However, the RPKM for oleosin-1 was far higher than that for oleosin-2, steroleosin, or caleosin (Figure 5), which was very similar to the temporal patterns in developing oilseeds of Brassica napus [16].

\section{Detection of SASK transcription factors involved in oil synthesis}

In previous studies, the transcription factors (TFs) such as ABI3, LEC1, LEC2, FUS3, WRI1, PKL, FIE, or GL2 play the key roles in seed oil synthesis and deposition [65]. To gain an insight into transcriptional regulatory mechanism of SASK oil biosynthesis by TFs, we analyzed all the unigenes of developing SASK by BLASTX against Arabidopsis TFs. The resulting 25 unigenes at developing SASK were identified as 9 homologies of ABI3 (AT3G24650), WRI1 (AT3G54320), LEC2 (AT1G28300), FUS3 (AT3G26790), ADOF1 (AT1G51700), PKL (AT2G25170), AP2 (AT4G36920), GL2 (AT1G79840), and HSI2 (AT2G30470) in Arabidopsis, but none of these 


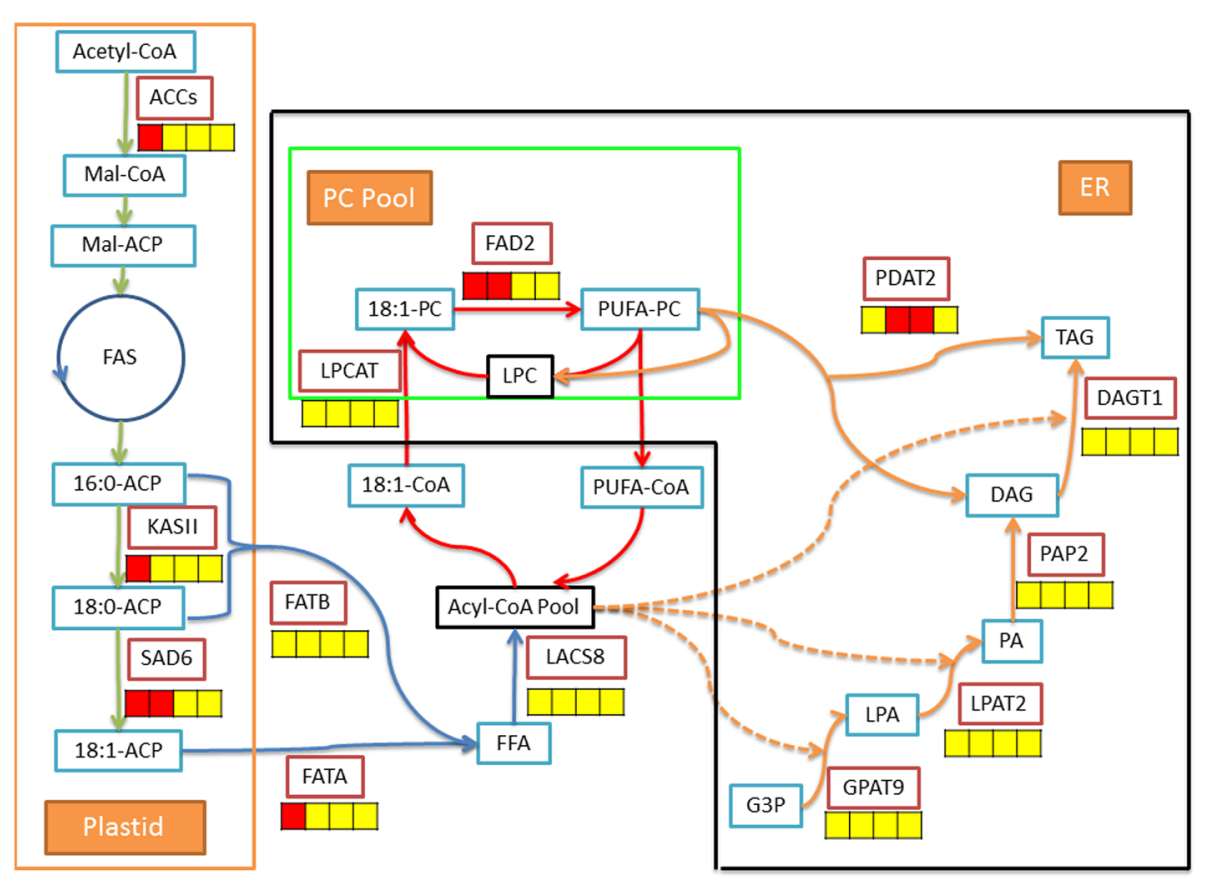

Figure 6 The temporal patterns for enzymes involved in oil synthesis. The icons below each enzyme show the result of DESeq analysis, from left to right: 10:30DAF, 10:50DAF, 10:60DAF, and 10:70DAF; red, up-regulation; yellow, no significant difference; green, down-regulation. Substrate abbreviations: ACP, acyl carrier protein; DAG, diacylglycerol; FFA, free fatty acid; G3P, glycerol-3-phosphate; LPA, lyso-phosphatidic acid; PA, phosphatidic acid; PC, phosphatidylcholine; PUFA, polyunsaturated fatty acids; TAG, triacylglycerol. Enzymatic abbreviations: ACC, acetyl-CoA carboxylase; DAG cholinephosphotransferase; DGAT1, acyl-COA:DAG acyltransferase 1; FAD2, fatty acid desaturase 2; FATA/B, acyl-ACP thioesterase A/B; GPAT9, acyl-COA:G3P acyltransferase 9; KASII, ketoacyl-ACP synthase II; LACS8, long chain acyl-CoA synthetase 8; LPAT2, acyl-CoA:LPA acyltransferase 2; LPCAT, acyl-CoA: lysophosphatidylcholine acyltransferase; PAP, PA phosphatase; PDAT2, phospholipid:DAG acyltransferase 2; PDCT: PC:DAG cholinephosphotransferase; SAD6, Stearoyl-ACP desaturase 6.

unigenes showed homology to LEC1, FIE, EMF2, or SWN (Additional file 10: Table S5). Moreover, our transcriptional profiling revealed that ABI3, WRI1, and LEC2 participated in positive regulation of the genes for oil synthesis in developing SASK, while AP2 and GL2 acted as negative regulatory factors. It is noteworthy that WRI1, the AP2domain-containing transcription factor, is known to be upregulated by LEC1, LEC2, ABI3, and FUS3 in Arabidopsis [66]. In our experiments, both FUS3 and WRI1 were shown with a high similar transcriptional profile in developing SASK (Figure 7), suggesting that the WRI1 gene might be a direct target of FUS3 in developing SASK. Additionally, the fact that plastidial $p k, p p t 1, p d c$, and $a c c$ ( $a c c A$ and $a c c C$ ) displayed a coordinated transcriptional profile with wri1 in developing SASK (Figures 5 and 7) indicated an involvement of WRI1 in the transcriptional regulation of target genes, as with Arabidopsis seeds [66]. Thus, SASK-WRI1 may play a contextual role in regulatory network of oil accumulation in developing SASK.

\section{Experimental validation and analysis of key genes involved in SASK oil accumulation}

To validate RPKM data, the expression profiles for $a c c C$, fat $B$, fat $A$, dgat1, and pdat 2 related to oil biosynthesis in developing SASK were analyzed by using qRT-PCR (Table 3). Our results showed that the relative expression levels of these genes were mostly consistent with the RPKM comparative ratios (10 DAF as the control). However, only dgat1 gene, not obtained further by DESeq method (Figure 6), was detected with significantly up-regulated expression in developing SASK by qRT-PCR analysis (Figure 8). Thus, differential expression analysis of SASK unigenes by DESeq method was reliable in this study.

It was hypothesized that the temporal transcriptional patterns for the genes involved in the biosynthesis of FAs and TAGs are very different in developing seed [16], which has been confirmed in our qRT-PCR results that $a c c C$ and $f a t A$ for FA synthesis, and dgat1 and pdat 2 for TAG formation in SASK were identified with a high expression at 10 to $30 \mathrm{DAF}$ and 50 to $70 \mathrm{DAF}$, respectively (Figure 8). This appears to indicate the beginning synthesis of the FAs and TAGs before 30 DAF and after 50 DAF, respectively. It was reported that dgat or pdat mutation had minimal impact on oil content in Arabidopsis seeds, showing that the overlapping functions of PDAT and DGAT are essential for TAG biosynthesis [62]. Notably, our investigations on the maximum expressed 


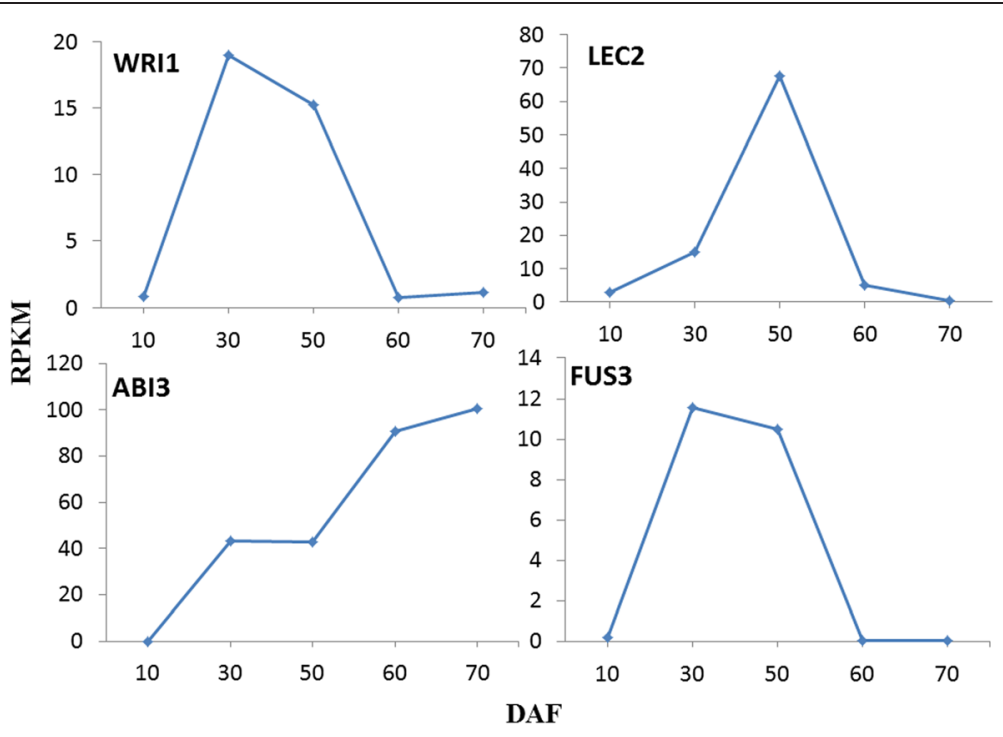

Figure 7 Temporal pattern of RPKM for SASK transcription factors.

value of pdat 2 and dgat 1 at 50 and 60 DAF respectively revealed an alteration of TAG accumulation in developing SASK by PDAT2 though PC pool or by DGAT1 via Kennedy pathway. All the above results evidenced that FAs and TAGs biosynthesis in developing SASK possessed complex dynamic regulation mechanisms, which deserve further research.

\section{Conclusions}

The Siberian apricot, a potential woody oil species for biodiesel, has a high quality and quantity of oil and excellent adaptability to different growing conditions. To fully understand temporally accumulated patterns of oil content and FA compositions, seven samples from the whole developmental SASK were firstly detected and analyzed. As a result, 60 DAF were characterized as the best time to obtain the high quality and quantity of SASK oil, and 5 experimental samples from the representative periods $(10,30,50,60$, and $70 \mathrm{DAF})$ were selected for comparative deep transcriptome analysis. Using the Illumina sequencing technology and Trinity program, 124,070 unigenes (mean length $=829.62 \mathrm{bp}$ ) were obtained from clean reads assembly and annotated in a public database, which could massively fill and enrich the dataset of Siberian apricot. There were 3,000, $2,781,2,620$, and 2,675 differentially expressed unigenes identified at 30, 50, 60, and 70 DAF using DESeq method (10 DAF as control), respectively. To reveal the accumulated mechanism of oil in developing SASK, the transcriptional profiles for the crucial transcription factors and metabolic enzymes involving in the biosynthesis of acetyl-CoA, FA, TAG, and oil body were systematically analyzed. We characterized that two transcription factors (WRI1 and FUS3) and some regulatory enzymes (PPT1, ptPK, ptPDC, ACC, KASII, SAD6, FATA, LACS8, FAD2, GPAT9, LPAT2, DGAT1, and PDAT2) play an important role in oil accumulation of developing SASK. Moreover, we also validated the temporal expression levels of five key genes (accC, fatB, fatA, dgat1, and pdat2) by qRT-PCR in developing SASK. All of these findings will be conducive to provide a substantial molecular biology background for researches on breeding, genetic diversity, and gene excavation of Siberian apricot and will promote the significant development for woody

Table 3 Genes and primer sequences used for real-time PCR

\begin{tabular}{llllll}
\hline Abbreviation & Gene name & $\begin{array}{l}\text { Accession } \\
\text { number }\end{array}$ & Primer forward & Primer reverse & $\begin{array}{l}\text { Amplicon } \\
\text { size (bp) }\end{array}$ \\
\hline accC & $\begin{array}{l}\text { Acetyl-CoA carboxylase. biotin } \\
\text { carboxylase subunit }\end{array}$ & KM364594 & CCAGGAAGAATAACCGCCTAC & GGGAGTCATAGTTGGAGGAC & 100 \\
fatB & Fatty acyl-ACP thioesterase B & KM364595 & GGCCCAAATATGCCAACAATC & GACGATCCATCCAGCCTTAAA & 97 \\
fatA & Fatty acyl-ACP thioesterase A & KM364596 & TGATGTCGGGAAGAGCATTAA & GGAGCAGGATCTTCCAGTTAG & 111 \\
dgat1 & Diacylglycerol acyltransferase 1 & KM364597 & CAGCCTATGTGTCTGCTTCTAT & CTCGATCCACACCTTGAGATT & 98 \\
pdat2 & $\begin{array}{l}\text { Phospholipid:diacylglycerol } \\
\text { acyltransferase 2 }\end{array}$ & KM364598 & TGATGACAGTGTGCCTGTATTG & CTTGTGCTGGTACTCCCTTATG & 115 \\
\hline
\end{tabular}




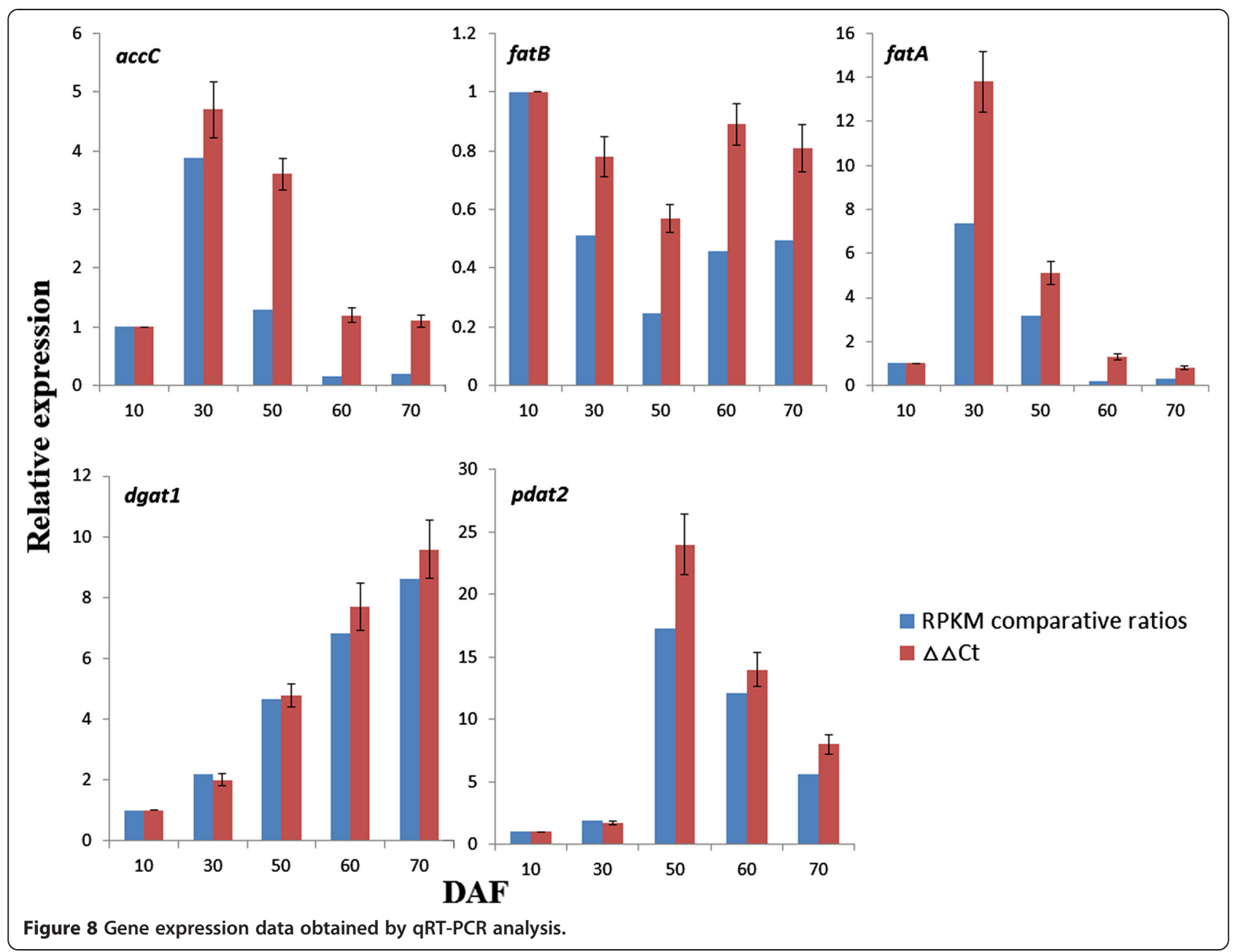

biodiesel. Certainly, we only analyzed a small subset of the mass data during SASK development and anticipate that other researchers would find valuable information from the SASK datasets available on request.

\section{Materials and methods}

\section{Plant materials and extraction of SASK Oil}

Siberian apricot is widely distributed in China, so it has not been listed as an endangered or protected species and does not require approval. The different developmental stages of SASK were obtained from the same tree located at the Beijing Forestry University experimental station, Beijing, China. The developmental processes of SASK from flowering to seed maturity were observed from May to July 2012. Flowers with the same anthesis were marked, and then seeds were harvested at $10 \mathrm{DAF}$ (immature stage), 20 DAF, 30 DAF, 40 DAF, 50 DAF, 60 DAF, and 70 DAF (fully matured stage), respectively. After removing the sarcocarp, the seeds were immediately frozen in liquid nitrogen and stored at $-80^{\circ} \mathrm{C}$ until use. The oil content of SASK and FA composition was determined using the previously described method [7].
CDNA Library preparation and sequence data analysis and assembly

According to the detective results of SASK oil content and FA composition, the experimental SASK from the five crucial periods $(10,30,50,60$, and $70 \mathrm{DAF})$ were selected as materials for comparative deep transcriptome and differential gene expression analysis. The equal weight of three biological samples from every developmental stage was mixed, and then total RNA was extracted from the mixture using RNeasy Plant Mini Kits (Qiagen, Inc., Valencia, CA, USA) according to the manufacturer's protocol. Extracted RNA was qualified and quantified using a Nanodrop ND-1000 Spectrophotometer (Nanodrop Technologies, Wilmington, DE, USA), and all the samples showed a $260 / 280 \mathrm{~nm}$ ratio from 1.9 to 2.1; no sign of degradation was found. The samples for transcriptome analysis were prepared using Illumina's kit following manufacturer's protocol (Illumina, San Diego, CA, USA). Briefly, the poly-(A) mRNA was purified from the total RNA by Oligotex mRNA Mini Kit (Qiagen, Inc., Valencia, CA, USA) following the manufacturer's protocol. cDNA library construction and 
normalization were performed using protocols described previously [67].

The cDNA library (two independent biological replicates) was sequenced on the Illumina sequencing platform (HiSeqTM 2000). After removal of the adapter sequences, the low-quality sequences (reads with ambiguous bases ' $\mathrm{N}$ ') and reads with more than $10 \% \mathrm{Q}<20$ bases, the quality of the clean reads were assessed by comparing the randomly selected 500,000 reads from all data to NT database with an E-value cut-off of $10^{-10}$ and coverage more than $80 \%$. Subsequently, the clean reads were assembled into unigenes with the Trinity program [25].

\section{Sequence annotation}

To understand their functions, the Siberian apricot unigenes were annotated using BLASTX alignment with an E-value cut-off of $10^{-5}$ against the following protein databases: NCBI nonredundant, Arabidopsis proteome, SwissProt, and eukaryotic orthologous groups. GO functional classifications and KEGG pathway assignments were performed, as was described previously and a detailed description of the workflow and settings used in the data analysis is given in [27].

\section{Differential expression analysis of unigenes}

The unigenes expression levels were calculated using reads per kilobase transcriptome per million mapped reads (RPKM), which could eliminated the influence of gene length and sequencing level on the calculation of gene expression. The levels of unigenes expression in the different samples were compared using the DESeq method described in [31].

\section{qRT-PCR validation}

Total RNA was extracted as the description for the cDNA library preparation and was reverse transcribed using the Reverse transcription System (Promega). The amplification primers were designed using PrimerQuest (http://www.idtdna.com/PrimerQuest/Home/Index) software with melting temperatures at $62^{\circ} \mathrm{C}$, and the absence of secondary structures was verified by the UNAFold program (http://eu.idtdna.com/UNAFold) according to D'haene et al. [68]. According to our previous studies, cyclophilin gene and ubiquitin-conjugating enzyme gene were used as an internal control [69]. The qRT-PCR was performed using the SYBR Premix Ex Taq Kit (TaKaRa) according to the manufacturer's protocol. Negative controls consisting of nuclease-free water instead of template, and reverse transcriptase controls prepared by substituting reverse transcriptase for nuclease-free water in the cDNA synthesis step were included in all analyses for each primer pair. Three technical repetitions were performed for qRT-PCR.

\section{Additional files}

Additional file 1: Table S1. Summary of SASK sequencing and
assembly.

Additional file 2: Figure S1. Length distribution of the unigenes. Additional file 3: Table S2. All unigenes' individual annotations, nt length, and levels of transcripts in RPKM.

Additional file 4: Table S3. Detailed information of the annotated genes based on Arabidopsis proteome.

Additional file 5: Figure S2. Histogram presentation of Gene Ontology classification. The results are summarized in three main categories: biological process, cellular component, and molecular function. The $y$-axis on the top indicates the number of genes, and the $y$-axis on the blow means the percent of genes in a category.

Additional file 6: Figure S3. COG classification. A total of 17,870 unigenes were assigned to 25 classifications. The capital letters in $\mathrm{x}$-axis indicates the COG categories as listed on the right of the histogram, and the $y$-axis indicates the number of unigenes.

Additional file 7: Figure S4. RPKM distribution of unigene.

Additional file 8: Figure S5. Comparative unigene expression profiles of five SASK developmental periods.

Additional file 9: Table S4. All up- or down-regulated genes and function annotations in distinct developing SASK by DESeq analysis.

Additional file 10: Table S5. The prediction of transcription factors involving in oil biosynthesis based on Arabidopsis protein.

\section{Abbreviations}

acetyl-CoA: acetyl-coenzyme A; ACC: acetyl-CoA carboxylase; ACCA: ACC carboxyl transferase subunit alpha; ACCC: ACC biotin carboxylase subunit; ACP: acyl-carrier protein; ACS: acetyl-CoA synthetase; AP: Arabidopsis proteome; BLAST: Basic Local Alignment Search Tool; COG: clusters of orthologous groups; DAF: days after flowering; DAG: diacylglycerol; DGAT: acyl-COA:DAG acyltransferase; EAR: enoyl-ACP reductase;

ER: endoplasmic reticulum; FA: fatty acid; FAD2: omega-6 FA desaturase; FATA: fatty acyl-ACP thioesterase A; FATB: fatty acyl-ACP thioesterase B; GLT1: glycolipid transporter; GO: gene ontology; G3P: glycerol-3-phosphate; GPAT: sn-1 G3P acyltransferase; GPT2: glucose-6-phosphate transporter; KAR: 3-ketoacyl ACP reductase; KASII: 3-ketoacyl ACP synthase II; KASIII: 3ketoacyl ACP synthase II; KEGG: Kyoto Encyclopedia of Genes and Genomes; LPAT: lysophosphatidyl acyltransferase; LACS: long-chain acyl CoA synthetase; LPCAT: acyl-CoA:lysophosphatidylcholine acyltransferase; MAT: malonyl-CoAACP transacylase; Nr: NCBI non-redundant protein; PA: phosphatidic acid; PAP: PA phosphohydrolases; PC: phosphatidylcholine; PDC: pyruvate dehydrogenase complex; PDAT: phospholipid: diacylglycerol acyltransferase; PDCT: PC:DAG cholinephosphotransferase; PFK: ATP-dependent phosphofructokinase; PFP: pyrophosphate-dependent phosphofructokinase; PK: pyruvate kinase; PPT1: phosphoenolpyruvate transporter; qRT-PCR: quantitative real-time PCR; RNA-Seq: RNA sequencing; SAD: 18:0-ACP desaturase; SASK: Siberian apricot seed kernels; SRA: Short Read Archive; SwissProt: Swiss-Prot protein database; TAG: triacylglycerol; TCA cycle: tricarboxylic acid cycle; TPT: triose phosphate transporter.

Competing interests

The authors declare that they have no competing interests.

\section{Authors' contributions}

$J \mathrm{~N}$ and JA organized and planned the research, and drafted the manuscript. $\mathrm{SZ}, \mathrm{ClF}$ and $\mathrm{HY}$ performed the sequence analysis. LW, XF, ZX, and XH determined the oil content and FA composition. LQ, CyF, DH, and HZ prepared the materials and CDNA samples. ZZ and SL provided funding, computational guidance, and were substantially involved in research design, data analysis, and manuscript preparation. All authors have read and approved the manuscript. 


\section{Acknowledgements}

This research was supported by the National Natural Sciences Foundation of China (No. J1103516), and the CentralPublic-Interest Scientific Institution Basal Research Fund (RIF2013-02) (No. 201004001).

\begin{abstract}
Author details
${ }^{1}$ College of Biological Sciences and Biotechnology, College of Nature Conservation, National Engineering Laboratory for Tree Breeding, Key Laboratory of Genetics and Breeding in Forest Trees and Ornamental Plants, Ministry of Education, Beijing Forestry University, Beijing 10083, China. ${ }^{2}$ Research Institute of Forestry, Chinese Academy of Forestry, Beijing 10091, China. ${ }^{3}$ Jigongshan National Nature Reserve, Xingyang 464133, China. ${ }^{4}$ Liaocheng Food and Drug Administration, Liaocheng 252000, Shandong, China.
\end{abstract}

Received: 1 September 2014 Accepted: 27 January 2015 Published online: 22 February 2015

\section{References}

1. Ghadge SV, Raheman H. Biodiesel production from mahua (Madhuca indica) oil having high free fatty acids. Biomass Bioenergy. 2005;28:601-5.

2. Lin SF, Shen Y. Thinking about the wood-oil biodiesel industry. Issues of Forestry Econ. 2010;30:131-5.

3. Wang LB, Yu HY, He XH, Liu RY. Influence of fatty acid composition of woody biodiesel plants on the fuel properties. J Fuel Chem Technol. 2012:40:397-404.

4. Wang LB. Resource investigation and distribute regular of three Armeniaca species. Forest Resource Manage. 2011;5:65-70.

5. Zhang JY, Wei ZZ, Zhao HL. Determination of ability of several arbor and shrub species to endure and survive extreme aridity with limited-areas methods under field conditions in Horqin Sandy Land. Acta Ecol Sin. 2006;26:467-74.

6. Wang LB. Geographic distribution and botanical characters of 3 Armeniaca plant in China. For Res. 2010;3:435-9.

7. Wang LB. Properties of Manchurian apricot (Prunus mandshurica Skv.) and Siberian apricot (Prunus sibirica L.) seed kernel oils and evaluation as biodiesel feedstocks. Ind Crop Prod. 2013;50:838-43.

8. Zhang JY. Chinese Fruit Species. Volume apricot. Beijng: China Forestry Publishing House; 2003. p. 627.

9. Wang LB. Evaluation of Siberian Apricot (Prunus sibirica L.) germplasm variability for biodiesel properties. J Am Oil Chem Soc. 2012:89:1743-7.

10. Wang LB, Yu HY. Biodiesel from Siberian apricot (Prunus sibirica L.) seed kernel oil. Bioresour Technol. 2012;112:355-8.

11. Wang LB, Chu J. Optimization of biodiesel production from Siberian Apricot (Prunus sibirica L.) oil using response surface methodology. Asian J Chem. 2013;25:2577-82

12. Dillies MA, Rau A, Aubert J, Hennequet-Antier C, Jeanmougin M, Servant N, et al. A comprehensive evaluation of normalization methods for Illumina high-throughput RNA sequencing data analysis. Brief Bioinform. 2012;14:671-83.

13. Huang JY, Jie ZJ, Wang $L$, Yan XH, Wei WH. Analysis of the differential expression of the genes related to Brassica napus seed development. Mol Biol Rep. 2011;38:1055-61.

14. Chen H, Wang FW, Dong YY, Wang N, Sun YP, Li XY, et al. Sequence mining and transcript profiling to explore differentially expressed genes associated with lipid biosynthesis during soybean seed development. BMC Plant Biol. 2012;12:122

15. Yin DM, Wang Y, Zhang XG, Li HM, Lu X, Zhang JS, et al. De novo assembly of the peanut (Arachis hypogaea L.) seed transcriptome revealed candidate unigenes for oil accumulation pathways. PLOS ONE. 2013;8:e73767.

16. Troncoso-Ponce MA, Kilaru A, Cao X, Durrett TP, Fan J, Jensen JK, et al. Comparative deep transcriptional profiling of four developing oilseeds. Plant J. 2011;68:1014-27.

17. Kroon J, Wei W, Simon WJ, Slabas AR. Identification and functional expression of a type 2 acyl-CoA: diacylglycerol acyltransferase (DGAT2) in developing castor bean seeds which has high homology to the major triglyceride biosynthetic enzyme of fungi and animals. Phytochemistry. 2006;67:2541-9.

18. Tao $X$, Fang $Y$, Xiao $Y$, Jin $Y L, M a ~ X R$, Zhao $Y$, et al. Comparative transcriptome analysis to investigate the high starch accumulation of duckweed (Landoltia punctata) under nutrient starvation. Biotechnol Biofuels. 2013;6:72

19. Bourgis F, Kilaru A, Cao X, Ngando-Ebongue G-F, Drira N, Ohlrogge JB, et al. Comparative transcriptome and metabolite analysis of oil palm and date palm mesocarp that differ dramatically in carbon partitioning. Proc Natl Acad Sci U S A. 2011;108:12527-32.

20. Fan HK, Xiao Y, Yang YD, Xia W, Mason AS, Xia ZH, et al. RNA-Seq analysis of Cocos nucifera: transcriptome sequencing and de novo assembly for subsequent functional genomics approaches. PLoS ONE. 2013;8:e59997.

21. Dong SB, Liu YL, Niu J, Ning Y, Lin SZ, Zhang ZX. De novo transcriptome analysis of the Siberian apricot (Prunus sibirica L.) and search for potential SSR markers by 454 pyrosequencing. Gene. 2014;544:220-7.

22. Wang R, Hanna MA, Zhou WW, Bhadury PS, Chen Q, Song BA, et al. Production and selected fuel properties of biodiesel from promising non-edible oils: Euphorbia lathyris L., Sapium sebiferum L. and Jatropha curcas L. Bioresour Technol. 2011;102:1194-9.

23. Lai ZX, Lin YL. Analysis of the global transcriptome of longan (Dimocarpus longan Lour.) embryogenic callus using Illumina paired-end sequencing. BMC Genomics. 2013;14:561.

24. Martin J, Bruno VM, Fang Z, Meng X, Blow M, Zhang T, et al. Rnnotator: an automated de novo transcriptome assembly pipeline from stranded RNA-Seq reads. BMC Genomics. 2010;11:663.

25. Grabherr MG, Haas BJ, Yassour M, Levin JZ, Thompson DA, Amit I, et al. Full-length transcriptome assembly from RNA-Seq data without a reference genome. Nat Biotechnol. 2011;29:644-52.

26. Sangwan RS, Tripathi S, Singh J, Narnoliya LK, Sangwan NS. De novo sequencing and assembly of Centella asiatica leaf transcriptome for mapping of structural, functional and regulatory genes with special reference to secondary metabolism. Gene. 2013;525:58-76.

27. Liu YL, Huang ZD, Ao Y, Li W, Zhang ZX. Transcriptome analysis of Yellow Horn (Xanthoceras sorbifolia Bunge.): a potential oil-rich seed tree for biodiesel in China. PLoS ONE. 2013:8:e74441.

28. Consortium GO. The Gene Ontology (GO) database and informatics resource. Nucleic Acids Res. 2004;32:D258-61.

29. Tatusov RL, Fedorova ND, Jackson JD, Jacobs AR, Kiryutin B, Koonin EV, et al. The COG database: an updated version includes eukaryotes. BMC Bioinformatics. 2003;4:41.

30. Kanehisa M, Goto S. KEGG: kyoto encyclopedia of genes and genomes. Nucleic Acids Res. 2000;28:27-30.

31. Anders S, Huber W. Differential expression of RNA-Seq data at the gene level-the DESeq package. Heidelberg, Germany: European Molecular Biology Laboratory (EMBL); 2012. Available at http://watson.nci.nih.gov/bioc_mirror/ packages/2.11/bioc/vignettes/DESeq/inst/doc/DESeq.pdf.

32. Mortazavi A, Williams BA, McCue K, Schaeffer L, Wold B. Mapping and quantifying mammalian transcriptomes by RNA-Seq. Nat Methods. 2008;5:621-8.

33. Rawsthorne S. Carbon flux and fatty acid synthesis in plants. Prog Lipid Res. 2002;41:182-96.

34. Andriotis VM, Kruger NJ, Pike MJ, Smith AM. Plastidial glycolysis in developing Arabidopsis embryos. New Phytol. 2010;185:649-62.

35. Plaxton WC. The organization and regulation of plant glycolysis. Annu Rev Plant Biol. 1996;47:185-214.

36. Weber AP, Schwacke R, Flügge U-I. Solute transporters of the plastid envelope membrane. Annu Rev Plant Biol. 2005;56:133-64.

37. Knappe S, Löttgert T, Schneider A, Voll L, Flügge UI, Fischer K. Characterization of two functional phosphoenolpyruvate/phosphate translocator (PPT) genes in Arabidopsis-AtPPT1 may be involved in the provision of signals for correct mesophyll development. Plant J. 2003;36:411-20.

38. Fatland BL, Ke J, Anderson MD, Mentzen WI, Cui LW, Allred CC, et al. Molecular characterization of a heteromeric ATP-citrate lyase that generates cytosolic acetyl-coenzyme A in Arabidopsis. Plant Physiol. 2002;130:740-56.

39. Ke J, Behal RH, Back SL, Nikolau BJ, Wurtele ES, Oliver DJ. The role of pyruvate dehydrogenase and acetyl-coenzyme A synthetase in fatty acid synthesis in developing Arabidopsis seeds. Plant Physiol. 2000;123:497-508

40. Randall DD, Miernyk JA, Fang TK, Budde RJ, Schuller KA. Regulation of the pyruvate dehydrogenase complexes in plants. Ann N Y Acad Sci. 1989;573:192-205.

41. Nikolau BJ, Ohlrogge JB, Wurtele ES. Plant biotin-containing carboxylases. Arch Biochem Biophys. 2003;414:211-22 
42. Bourrellier ABF, Valot B, Guillot A, Ambard-Bretteville F, Vidal J, Hodges M. Chloroplast acetyl-CoA carboxylase activity is 2-oxoglutarate-regulated by interaction of PII with the biotin carboxyl carrier subunit. Proc Natl Acad Sci U S A. 2010;107:502-7.

43. Andre C, Haslam RP, Shanklin J. Feedback regulation of plastidic acetyl-CoA carboxylase by 18: 1-acyl carrier protein in Brassica napus. Proc Natl Acad Sci U S A. 2012;109:10107-12.

44. Bates PD, Stymne S, Ohlrogge J. Biochemical pathways in seed oil synthesis. Curr Opin Plant Biol. 2013;16:358-64.

45. Cahoon EB, Clemente TE, Damude HG, Kinney AJ. Oil Crops Handbook of Plant Breeding (Modifying Vegetable Oils for Food and Non-Food Purposes). Volume 4. New York: Springer; 2010. p. 31-56.

46. Voelker TA, Hayes TR, Cranmer AM, Turner JC, Davies HM. Genetic engineering of a quantitative trait: metabolic and genetic parameters influencing the accumulation of laurate in rapeseed. Plant J. 1996;9:229-41.

47. Knutzon DS, Thompson GA, Radke SE, Johnson WB, Knauf VC, Kridl JC. Modification of Brassica seed oil by antisense expression of a stearoyl-acyl carrier protein desaturase gene. Proc Natl Acad Sci U S A. 1992;89:2624-8.

48. Pidkowich MS, Nguyen HT, Heilmann I, Ischebeck T, Shanklin J. Modulating seed $\beta$-ketoacyl-acyl carrier protein synthase II level converts the composition of a temperate seed oil to that of a palm-like tropical oil. Proc Natl Acad Sci U S A. 2007;104:4742-7.

49. Koo AJ, Ohlrogge JB, Pollard M. On the export of fatty acids from the chloroplast. J Biol Chem. 2004;279:16101-10.

50. Zhao LF, Katavic V, Li FL, Haughn GW, Kunst L. Insertional mutant analysis reveals that long-chain acyl-CoA synthetase 1 (LACS1), but not LACS8, functionally overlaps with LACS9 in Arabidopsis seed oil biosynthesis. Plant J. 2010;64:1048-58.

51. Behrouzian B, Buist PH. Fatty acid desaturation: variations on an oxidative theme. Curr Opin Chem Biol. 2002;6:577-82.

52. Kim S, Yamaoka Y, Ono H, Kim H, Shim D, Maeshima M, et al. AtABCA9 transporter supplies fatty acids for lipid synthesis to the endoplasmic reticulum. Proc Natl Acad Sci U S A. 2013;110:773-8.

53. Barron EJ, Stumpf P. Fat metabolism in higher plants XIX. The biosynthesis of triglycerides by avocado-mesocarp enzymes. Biochim Biophys Acta. 1962;60:329-37.

54. Yang W, Simpson JP, Li-Beisson Y, Beisson F, Pollard M, Ohlrogge JB. A land-plant-specific glycerol-3-phosphate acyltransferase family in Arabidopsis: substrate specificity, sn-2 preference, and evolution. Plant Physiol. 2012;160:638-52.

55. Gidda SK, Shockey JM, Rothstein SJ, Dyer JM, Mullen RT. Arabidopsis thaliana GPAT8 and GPAT9 are localized to the ER and possess distinct ER retrieval signals: Functional divergence of the dilysine ER retrieval motif in plant cells. Plant Physiol Biochem. 2009;47:867-79.

56. Maisonneuve S, Bessoule J-J, Lessire R, Delseny M, Roscoe TJ. Expression of rapeseed microsomal lysophosphatidic acid acyltransferase isozymes enhances seed oil content in Arabidopsis. Plant Physiol. 2010;152:670-84.

57. Eastmond PJ, Quettier A-L, Kroon JT, Craddock C, Adams N, Slabas AR. PHOSPHATIDIC ACID PHOSPHOHYDROLASE1 and 2 regulate phospholipid synthesis at the endoplasmic reticulum in Arabidopsis. Plant Cell. 2010;22:2796-811.

58. Kim HU, Li Y, Huang AH. Ubiquitous and endoplasmic reticulum-located lysophosphatidyl acyltransferase, LPAT2, is essential for female but not male gametophyte development in Arabidopsis. Plant Cell. 2005;17:1073-89.

59. Lu C, Xin Z, Ren Z, Miquel M. An enzyme regulating triacylglycerol composition is encoded by the ROD1 gene of Arabidopsis. Proc Natl Acad Sci U S A. 2009;106:18837-42.

60. Saha S, Enugutti B, Rajakumari S, Rajasekharan R. Cytosolic triacylglycerol biosynthetic pathway in oilseeds. Molecular cloning and expression of peanut cytosolic diacylglycerol acyltransferase. Plant Physiol. 2006;141:1533-43.

61. Shockey JM, Gidda SK, Chapital DC, Kuan J-C, Dhanoa PK, Bland JM, et al. Tung tree DGAT1 and DGAT2 have nonredundant functions in triacylglycerol biosynthesis and are localized to different subdomains of the endoplasmic reticulum. Plant Cell. 2006;18:2294-313.

62. Zhang M, Fan J, Taylor DC, Ohlrogge JB. DGAT1 and PDAT1 acyltransferases have overlapping functions in Arabidopsis triacylglycerol biosynthesis and are essential for normal pollen and seed development. Plant Cell. 2009;21:3885-901.

63. Mhaske V, Beldjilali K, Ohlrogge J, Pollard M. Isolation and characterization of an Arabidopsis thaliana knockout line for phospholipid: diacylglycerol transacylase gene (At5g13640). Plant Physiol Biochem. 2005;43:413-7.
64. Jolivet P, Roux E, d'Andrea S, Davanture M, Negroni L, Zivy M, et al. Protein composition of oil bodies in Arabidopsis thaliana ecotype WS. Plant Physiol Biochem. 2004;42:501-9.

65. Palaniswamy SK, James S, Sun H, Lamb RS, Davuluri RV, Grotewold E. AGRIS and AtRegNet. a platform to link cis-regulatory elements and transcription factors into regulatory networks. Plant Physiol. 2006;140:818-29.

66. Baud S, Lepiniec L. Physiological and developmental regulation of seed oil production. Prog Lipid Res. 2010:49:235-49.

67. Xu Q, Zhao WM, Chen Y, Tong YY, Rong GH, Huang ZY, et al. Transcriptome profiling of the goose (Anser cygnoides) ovaries identify laying and broodiness phenotypes. PLOS ONE. 2013;8:e55496.

68. Løvdal T, Saha A. Reference gene selection in Carnobacterium maltaromaticum, Lactobacillus curvatus and Listeria innocua subjected to temperature and salt stress. Mol Biotechnol. 2014;56:210-22.

69. Niu J, Zhu BQ, Cai J, Li PX, Wang LB, Dai HT, et al. Selection of reference genes for gene expression studies in Siberian Apricot (Prunus sibirica L.) germplasm using Quantitative Real-Time PCR. PLoS ONE. 2014;9:e103900.

\section{Submit your next manuscript to BioMed Central and take full advantage of:}

- Convenient online submission

- Thorough peer review

- No space constraints or color figure charges

- Immediate publication on acceptance

- Inclusion in PubMed, CAS, Scopus and Google Scholar

- Research which is freely available for redistribution 